\section{Statistical analysis of comparative data}

Emilia Martins' TREE review ${ }^{1}$ of some recent developments in the field of comparative methods gives the impression that serious doubts have been raised regarding the validity of these methods for drawing inferences about adaptation. The current vitality of the field suggests otherwise. Statistical models applied to phylogenies are overturning many long-held beliefs about the history of life on earth ${ }^{2}$, including inferences about ancient character states, timings and evolutionary processes. The success of statistical models means that they have become serious independent forms of enquiry, which complement traditional palaeontological approaches to understanding the past.

Martins discusses several comparative method issues that have attracted attention over the years: ancestral state reconstructions, the assumptions of models and the necessity of using a phylogenetic approach in any given circumstance. Reconstructed ancestral states are sometimes found to have large standard errors, thus indicating uncertainty in the reconstruction. Some commentators interpret this uncertainty as problematic; however, what is a weakness to some is a virtue to others. It is only the statistical methods that, by estimating uncertainty ${ }^{2-4}$, call attention to the loss of history that is inevitable in any evolving process. By contrast, nonstatistical approaches give no hint that the character state they assign might not be substantially better supported than the alternatives. I do not suggest that Martins would disagree with this, but to say that 'it is too soon to dismiss the use of statistical [comparative

methods]...based solely on estimation accuracy' might lead less-experienced practitioners to draw the wrong conclusions.

Similar views often arise in response to the other two topics. Martins puts forward the usual defence of models of evolution stating that, restrictive as their assumptions might be, their use is usually better than doing nothing at all. I agree and wish to emphasize that a stronger position can be adopted. It is possible to use the data in combination with the phylogeny to estimate scaling parameters that optimally transform the branch lengths of the phylogeny to conform to the underlying assumptions of the model ${ }^{2,5}$. In effect, they find the branch-length basis in which trait evolution best matches the nature of evolution supposed by the model. The scaling can detect when the tempo and mode of evolution depart from the usual assumption of a constant-variance random walk, including punctuational change, adaptive radiations (the subject of the Price model ${ }^{6}$ that Martins discusses), species-specific adaptations and directional biases in evolution. It is even possible to assess and then scale the contribution of the phylogeny itself.

The amount of gene-sequence information is growing at rates thought impossible only a few years ago, thus reliable phylogenies with useful branch-length information are becoming commonplace for many taxonomic groups. Comparative methods are possibly better placed than ever to infer adaptive processes from these phylogenies.

\section{Mark Pagel}

School of Animal and Microbial Sciences, University of Reading, Reading, UK RG6 6AJ (m.pagel@reading.ac.uk)

\section{References}

1 Martins, E.P. (2000) Adaptation and the comparative method. Trends Ecol. Evol. 15, 296-299

2 Pagel, M. (1999) Inferring the historical patterns of biological evolution. Nature 401, 877-884

3 Schluter, D. et al. (1997) Likelihood of ancestor states in adaptive radiation. Evolution 51, 1699-1711

4 Garland, T., Jr and Ives, A.R. (2000) Using the past to predict the present: confidence intervals for regression equations in phylogenetic comparative methods. Am. Nat. $155,346-364$

5 Pagel, M. (1997) Inferring evolutionary processes from phylogenies. Zool. Scripta 26, 331-348

6 Price, T. (1997) Correlated evolution and independent contrasts. Philos. Trans. R. Soc. London Ser. B 352, 519-529

\section{Immunosuppression under stress: necessary for condition-dependent signalling?}

In a recent TREE review, Buchanan ${ }^{1}$ discussed the implications of stress for the evolution of condition-dependent signals. With regard to the immune system, the author suggests a new route by which the immunocompetence handicap hypothesis ${ }^{2}$ could function: genetic variation in immune traits might lead to different capabilities to manage stress-induced immunosuppression. This might be signalled through condition-dependent handicap traits. Buchanan ${ }^{1}$ emphasizes that this hypothesis requires that naturally occurring levels of stress hormones, such as corticosteroids, are immunosuppressive. However, the occurrence of such stress-induced immunosuppression has recently been questioned ${ }^{3}$.

Braude et al. ${ }^{3}$ suggested that physiological levels of corticosteroids might not really lead to immunosuppression but rather to immunoredistribution, which is a temporary shifting of immune power to components of the body where it is likely to be most useful. It would be easy to confuse immunoredistribution with immunosuppression if immunity is assessed by blood leukocyte counts only, for example. A similar mechanism was suggested for testosterone ${ }^{3}$, thus challenging the immunocompetence handicap hypothesis. If stress hormones and testosterone lead to immunoredistribution instead of immunosuppression, could conditiondependent signalling, based on variability in immunocompetence, still work? We argue that it might work with immunoredistribution as successfully as it does with immunosuppression, provided that immune

functions are somehow costly.

The immune system must be regulated precisely to enable pathogen elimination without autoimmunity or hypersensitivity. Immunoredistribution, as well as immunosuppression, might be the means to achieve these goals. Stress-response behaviour (e.g. escape flight or other heavy physical exercise) is often associated with muscle damage 4 and increased metabolic turnover, resulting in the formation of detrimental products (especially reactive oxygen intermediates) that have a nonspecific, damaging effect on tissue ${ }^{5,6}$. Because tissue breakdown leads to the presentation of new antigens, poststress immunosuppression was suggested to help avoid autoimmune reactions against these new antigens 7,8 . However, immune cells are needed to eliminate damaged tissue; redistribution of immune functions not involved in autoimmunity to the sites of damage would consequently be adaptive. In response to stress, the immune system might therefore shift its power to sites of muscle damage and oxidative stress. This immunoredistribution would actually result in apparent immunosuppression concerning parasites, because less immune power remains for parasite defence. Seen from this angle, immunoredistribution is probably an adaptive tradeoff within the immune system used to cope with different demands. This view of immunoredistribution is compatible with condition-dependent signalling: only individuals with well adapted immune genes might be able to manage the phenotypically reduced parasite defence resulting from immunoredistribution under stressful conditions.

Such a system would only work when individuals are unable to fully maintain both stress-related and parasite-related immune functions; that is, immunity has to be costly. Several recent reviews emphasize the strong effect of energy limitations on nearly all immune functions $1,9,10$. Under a life cycle perspective, maintaining defences in the absence of infection is costly, as shown for competitive ability in Drosophila larvae ${ }^{11}$. In summary, it seems reasonable to suppose that immunity is costly and that costs differ between individuals depending on their immune genes. Condition-dependent signalling based on stress-induced modulation of the immune system could therefore work, irrespective of whether stress leads to immunosuppression or immunoredistribution.

\section{Joachim Kurtz}

Max-Planck-Institut für Limnologie, Abteilung Evolutionsökologie, AugustThienemann-Str. 2, 24306 Ploen, Germany (kurtz@mpil-ploen.mpg.de)

\section{Klaus Reinhold Leif Engqvist}

Institut für Evolutionsbiologie und Ökologie, Rheinische Friedrich-WilhelmsUniversität Bonn, An der Immenburg 1, 53121 Bonn, Germany

(kreinhold@evolution.uni-bonn.de; lengqvist@evolution.uni-bonn.de) 


\section{References}

1 Buchanan, K.L. (2000) Stress and the evolution of condition-dependent signals. Trends Ecol. Evol. 15, 156-160

2 Folstad, I. and Karter, A.J. (1992) Parasites, bright males, and the immunocompetence handicap. Am. Nat. 139, 603-622

3 Braude, S. et al. (1999) Stress, testosterone, and the immunoredistribution hypothesis. Behav. Ecol. 10, 345-350

4 Hoffman-Goetz, L. and Pedersen, B.K. (1994) Exercise and the immune system: a model of the stress response? Immunol. Today 15, 382-387

5 Rose, M.R. (1991) Evolutionary Biology of Aging, Oxford University Press

6 Jenkins, R.R. (1993) Exercise, oxidative stress, and antioxidants: a review. Int. J. Sports. Nutr. 3, 356-375

7 Pedersen, B.K. et al. (1994) The immune system during exposure to extreme physiologic conditions. Int. J. Sports Med. 15, S116-S121

8 Råberg, L. et al. (1998) On the adaptive significance of stress-induced immunosuppression. Proc. R. Soc. London Ser. B 265, 1637-1641

9 Buttgereit, F. et al. (2000) Bioenergetics of immune functions: fundamental and therapeutic aspects. Immunol. Today 21, 192-199

10 Svensson, E. et al. (1998) Energetic stress, immunosuppression and the costs of an antibody response. Funct. Ecol. 12, 912-919

11 Kraaijeveld, A.R. and Godfray, H.C.J. (1997) Trade-off between parasitoid resistance and larval competitive ability in Drosophila melanogaster. Nature 389, 278-280

\section{Reply from K.L. Buchanan}

I welcome the comments made by Kurtz et al. ${ }^{1}$, which highlight the potential importance of immunoredistribution ${ }^{2}$ (as an alternative to immunosuppression) as a mechanism linking stress with condition-dependent signalling.

It seems possible that immunoredistribution could occur through two possible scenarios: resources could be shifted between different places in the body or resources could be shunted between different aspects of the immune system involved in specific types of defence. This is an important distinction because shifting resources relies on migration of preexisting cells, whereas the shunting resources scenario implies an intrinsic tradeoff in either resources or energy. Currently, the strongest evidence exists for the movement of immune resources within the body ${ }^{2}$. Either mechanism could control the development of condition-dependent traits through the genetically controlled ability to modulate the immune response efficiently. Demonstration that stress-linked immunoredistribution has relevance for the evolution of condition-dependent traits requires that: (1) commonly occurring stressful incidents cause a redirection of resources away from fighting pathogens or disease; and (2) such stressful incidents are relevant during the development of conditiondependent traits.
As reviewed by Braude et al. ${ }^{2}$, there is convincing evidence that relocation of resources can occur during an acute stress response. Futhermore, because the generalized nature of the stress response has been well documented ${ }^{3}$, it seems probable that similar immunoredistribution effects occur in response to a variety of stimuli. However, pathogens and disease can potentially attack the host on many fronts, and a coordinated immune response is therefore required to keep them under control.

Therefore, it would seem unwise to suggest that stress-linked immunoredistribution will always occur at the detriment of parasitic defence. For example, a bird ingesting a toxic fruit might develop an acute stress response, but this might or might not direct resources away from parasite defence. Currently, there is little evidence to suggest that there is sufficient specificity in the stress response for immunoredistribution to vary according to the stress stimuli. However, this might, in part, be due to a current lack of understanding of the subtle differences that might exist in the response to commonly experienced stressors ${ }^{4}$.

Although there is evidence that acute stress can cause leukocyte redistribution and an enhancement of certain immune responses ${ }^{2}$, there is considerable contrasting evidence that chronic elevations in corticosteroids cause immunosuppression of both cell-mediated and humoral functions ${ }^{5}$. This might, in part, be due to differences in the tradeoffs within the immune system and with other resources under different circumstances of stress. For example, it could be essential to heal a wound from a predatory attack, causing the mobilization of all available resources at the detriment of parasite defence. However, in the face of chronic stress, such as long-term food deprivation, the tradeoff might be quite different. Therefore, it is essential to understand the nature of the stress events commonly experienced and their relevance during the period when the conditiondependent trait is developing. If chronic stress is more biologically meaningful during the development of condition-dependent signals, then immunosuppression would be the more appropriate mechanism allowing the cost to be mediated.

\section{Katherine L. Buchanan}

Avian Ecology Unit, Dept of Biological Sciences, University of Stirling, Stirling, UK FK9 4LA (k.l.buchanan@stir.ac.uk)

\section{References}

1 Kurtz, J. et al. (2000) Immunosuppression under stress: necessary for condition-dependent signalling? Trends Ecol. Evol. 15, 418-419

2 Braude, S. et al. (2000) Stress, testosterone, and the immunoredistribution hypothesis. Behav. Ecol. 10, 345-350

3 Broom, D.M. and Johnsen, K.G. (1993) Stress and Animal Welfare, Chapman \& Hall

4 Saplosky, R.M (1992) Neuroendocrinology of the stress response. In Behavioural Endocrinology (Becker, J.B. et al., eds), pp. 287-324, Massachusetts Institute of Technology
5 Buchanan, K.L. (2000) Stress and the evolution of condition-dependent signals Trends Ecol. Evol. 15, 156-160

\section{Disruptive sexual selection}

In a recent TREE News \& Comment, Bridle and Jiggins ${ }^{1}$ discuss growing empirical support for sympatric speciation, and for putative mechanisms that might underlie its occurrence. They dwell primarily on disruptive natural selection, touching only fleetingly on how disruptive sexual selection could drive sympatric speciation via assortative mating, leading to divergent Fisherian runaway processes ${ }^{2}$. Two models have formally addressed disruptive sexual selection: our own in 1997 (Ref. 3) and a more recent model by Higashi et al. ${ }^{4}$ These models rely on different behavioural assumptions, but both are underpinned by a bifurcating Fisherian process. Thus, a comparison of the models might offer insight into factors essential to sympatric speciation by disruptive sexual selection.

The model by Higashi et al. considers linkage disequilibrium arising in genes coding for quantitative traits - a male signalling trait and a female preference trait. Females prefer males whose trait lies closer to either extreme; thus, female preferences are, in a sense, 'localized' in trait space. Spontaneous speciation occurs when the characteristic scale associated with the preferences is small relative to the width of the distribution of the male trait in the original unimodal population. Hence, females have only a negligible interest in males at the opposing extreme.

Our own model used major gene coding, but explicitly accounted for spatial dispersal, assuming that males unsuccessful in courtship move around more. Thus, female preferences are intrinsically localized in space. Unsuccessful males disperse and carry with them linked preference genes, so that neighbouring areas develop Fisherian runaway in the opposite direction to that in the original locale. There is a spontaneous polarization of opposing trait-preference linkage pairs in adjoining spatial regions, dependent on the spatial-scale characteristic of the individual dispersal or migratory behaviour.

Higashi et al. report that their model relies upon both trait extremes being equally favoured at the population level, with prezygotic isolation becoming established before one or other subpopulation is extinguished. Simulations of our own model indicate that the incipient spatial patterning is robust to even moderate asymmetries, with the boundary zone open to neutral drift ${ }^{3}$. Natural populations typically experience environmental heterogeneity in viability selection on male traits and this is likely to destabilize the process of Higashi et al. By contrast, such heterogeneity enhances fixation of the spatial position of the boundary zone in our model.

Thus, there is more than one route by which divergent Fisherian runaway can arise. In both models, it is the restricted 'localization' 